\title{
Identification of Hadronic Tau decays in ATLAS*
}

\author{
Y. Coadou ${ }^{a, b}$, I. Hinchliffe ${ }^{b}$, J. Lozano-Bahilo ${ }^{b}$, L.C. Loveridge ${ }^{b}$ M.D. Shapiro ${ }^{b}$ \\ ${ }^{a}$ Kungliga Tekniska Högskolan, Sweden \\ ${ }^{b}$ Lawrence Berkeley National Laboratory, Berkeley, CA
}

\begin{abstract}
Taus can be an important signature for Supersymmetry at the LHC. They can be produced copiously in the decays of supersymmetric particles. Measurement of their momentum and of the tau-tau invariant mass distribution, would provide detailed information regarding the masses of supersymmetric particles. It is demonstrated using full simulation that it will be possible for ATLAS to make a cut on the reconstructed tau invariant mass using information from the tracking and the electromagnetic calorimeter. The measured energy and momentum of tau's that pass this selection can then be used to infer information about the tau-tau invariant mass.
\end{abstract}

*This work was supported in part by the Director, Office of Energy Research, Office of High Energy and Nuclear Physics, Division of High Energy Physics of the U.S. Department of Energy under Contracts DE-AC03-76SF00098. 


\section{DISCLAIMER}

This report was prepared as an account of work sponsored by an agency of the United States Government. Neither the United States Government nor any agency thereof, nor any of their employees, make any warranty, express or implied, or assumes any legal liability or responsibility for the accuracy, completeness, or usefulness of any information, apparatus, product, or process disclosed, or represents that its use would not infringe privately owned rights. Reference herein to any specific commercial product, process, or service by trade name, trademark, manufacturer, or otherwise does not necessarily constitute or imply its endorsement, recommendation, or favoring by the United States Government or any agency thereof. The views and opinions of authors expressed herein do not necessarily state or reflect those of the United States Government or any agency thereof. 


\section{DISCLAIMER}

Portions of this document may be illegible in electronic image products. Images are produced from the best available original document. 


\section{Introduction}

The identification and measurement of tau's at the LHC is an important issue. Many new particles can decay into final states involving taus. Detailed studies [1] have been carried out for the case of a Higgs boson decaying to $\tau^{+} \tau^{-}$. If the Higgs boson is heavy, the decay products of the tau closely follow the tau's direction. The tau's energy cannot be measured directly as neutrinos in the tau decay carry off energy. However the total transverse momentum carried by all the neutrinos gives rise to missing transverse energy which can be measured by the ATLAS detector. This measurement, combined with a knowledge of the tau direction, is sufficient for the $\tau^{+} \tau^{-}$mass to be reconstructed on an event by event basis, the mass resolution being determined by the missing transverse energy resolution of the detector. [1] In this case the signal to background ratio is rather poor and stringent tau selection cuts must be applied. Events are selected by requiring one tau to decay leptonically and the other to decay to a single hadron. The large standard model background can be controlled and a signal extracted. [2]

Taus can also be produced as a decay products of supersymmetric particles in a large class of supersymmetric models. In most cases at the LHC, the production of supersymmetric particles is dominated by that of squarks $(\tilde{q})$ and gluinos $(\tilde{g})$ as they have strong interactions. These then decay into other supersymmetric particles, in particular into sleptons $(\tilde{\ell})$ and gauginos $(\tilde{\chi})$. A characteristic decay chain, valid over a large fraction of parameter space involves the following

$$
\tilde{q} \rightarrow q \tilde{\chi_{2}^{0}}\left(\rightarrow \ell \tilde{\ell}\left(\rightarrow \ell \tilde{\chi_{1}^{0}}\right)\right)
$$

which gives rise to a final state containing missing energy (carried off by $\tilde{\chi}_{1}^{0}$ )) and a dilepton pair. The invariant mass distribution of the dilepton pair has a sharp end point at

$$
M_{\ell \ell}^{\max }=M_{\tilde{\chi}_{2}^{0}} \sqrt{1-\frac{M_{\tilde{\ell}}^{2}}{M_{\tilde{\chi}_{2}^{0}}^{2}}} \sqrt{1-\frac{M_{\tilde{\chi}_{1}^{0}}^{2}}{M_{\tilde{\ell}}^{2}}}
$$

An example of this type is discussed in detail elsewhere; it was Point 5 of the original set of LHC supersymmetry studies [3,5]. Measurement of the position of this endpoint provides a precise measurement of a combination of masses and selection of events near this endpoint provides a starting point which allows reconstruction of the entire decay chain. In the cases where $\tilde{e}, \tilde{\mu}$ and $\tilde{\tau}$ are degenerate, the measurement can be carried out with precision in the $e$ and $\mu$ pair final states. However there is an important subclass of models where the parameter $\tan \beta$ is large, the sleptons are not degenerate, the $\tilde{\tau}$ is the lightest and the decays to $\tilde{e}$ and $\tilde{\mu}$ are not allowed.

It is necessary to identify and measure the taus. The technique discussed above cannot be used to reconstruct the $\tau-\tau$ invariant mass as there are particles in the event $\left(\tilde{\chi}_{1}^{0}\right)$ other than the neutrinos from the tau decays that contribute to missing energy. In this note, we describe a method for selecting hadronic tau decays in such a way that the energy carried by neutrinos is minimized and the end point of the tau-tau invariant mass distribution can be measured.

This study is based on full simulation of the ATLAS detector using DICE 97-6 [4]. It is impractical to simulate full supersymmetry events due to their complexity and the 
consequent CPU time required to generate a sufficient number of events. The $\tau \tau$ invariant mass distribution was therefore studied by generating a sample of $Z+$ jet events with the $Z$ 's forced to decay to $\tau \tau$. This has the advantage that the invariant mass of the $\tau \tau$ system is known. By varying the mass of the $Z$ in the generator, the reconstructed $\tau \tau$ invariant mass can be studied over the kinematic range relevant to the supersymmetry signal. No pile up is included in this study, whose conclusions are therefore limited to the case of low luminosity running (i.e. $10^{33} \mathrm{~cm}^{-2} \mathrm{sec}^{-1}$ ). We first describe a selection for hadronically decaying taus based on isolation in the electromagnetic calorimeter. The rejection factors against jets can be studied using the QCD jets in the same events. We then describe the reconstruction of the invariant mass of the visible tau decay products using a combination of the electromagnetic calorimeter and the tracking system. Using a cut based on this quantity we reconstruct the $\tau \tau$ invariant mass. We finally provide a simple parameterization of our results that can be used in a fast simulation of the supersymmetric case.

\section{Tau selection and isolation}

Pythia 5.7 [6] and DICE 97-6 ${ }^{\dagger}$ was used to generate samples of $Z+j e t$ events and simulate them through the ATLAS detector. Each sample consisted of 500 events with $Z \rightarrow \tau \tau$ and $\tau \rightarrow$ hadrons being forced. Leptonic tau decays are not useful because, in them, there are two neutrinos and therefore the fraction of the tau energy carried by the lepton is small, and because there is no way to distinguish an e or $\mu$ produced in the decay of a $\tau$ from one produced directly. Samples were generated with $M_{Z}=91$ and for the transverse momentum of the $Z$ in ranges $50-75,125-150$ and $175-200 \mathrm{GeV}$. For the central $p_{t}$ range samples where also produced for $M_{Z}=25,50,75$, and $200 \mathrm{GeV}$. This produces taus over the same kinematic range as will be needed for the supersymmetry cases.

The hadronic jets from tau decays are distinguished from QCD jets by their lower particle multiplicity, narrower profile and smaller invariant mass. We will use these features to select taus and reject jets. Jets were found by using a fixed cone algorithm. The (electromagnetic and hadronic) calorimeter energy deposits were mapped onto a grid of $\Delta \phi \times \Delta \eta=0.1 \times 0.1$. A cluster finder using a cone of radius $\Delta R$ in $\eta \times \phi$ space was then used to find the jets which were retained if the total transverse energy in the cone was greater than $10 \mathrm{GeV}$. The full segmentation of the ATLAS calorimeter was then used to look at the detailed structure of the jets. Calorimetric isolation was then applied by studying the ratio of the energy in the outer region of a jet to that in the core defined by

$$
I=\frac{E(0.4)-E(0.2)}{E(0.2)}
$$

Where $E(\Delta R)$ is the energy inside a cone of radius $\Delta R$ in $\eta \times \phi$ space around the center of the jet. Figure 1 shows this distribution made from the energy measured in the electromagnetic calorimeter, which has finer segmentation.

The difference in shape of these two distributions is clear, the tau decays (top plot) having the narrower distribution. By rejecting events with $I>I_{0}$, an enriched tau sample can be

\footnotetext{
${ }^{\dagger}$ This version of DICE produces occasional events with large energy deposits. These events are removed from the sample.
} 


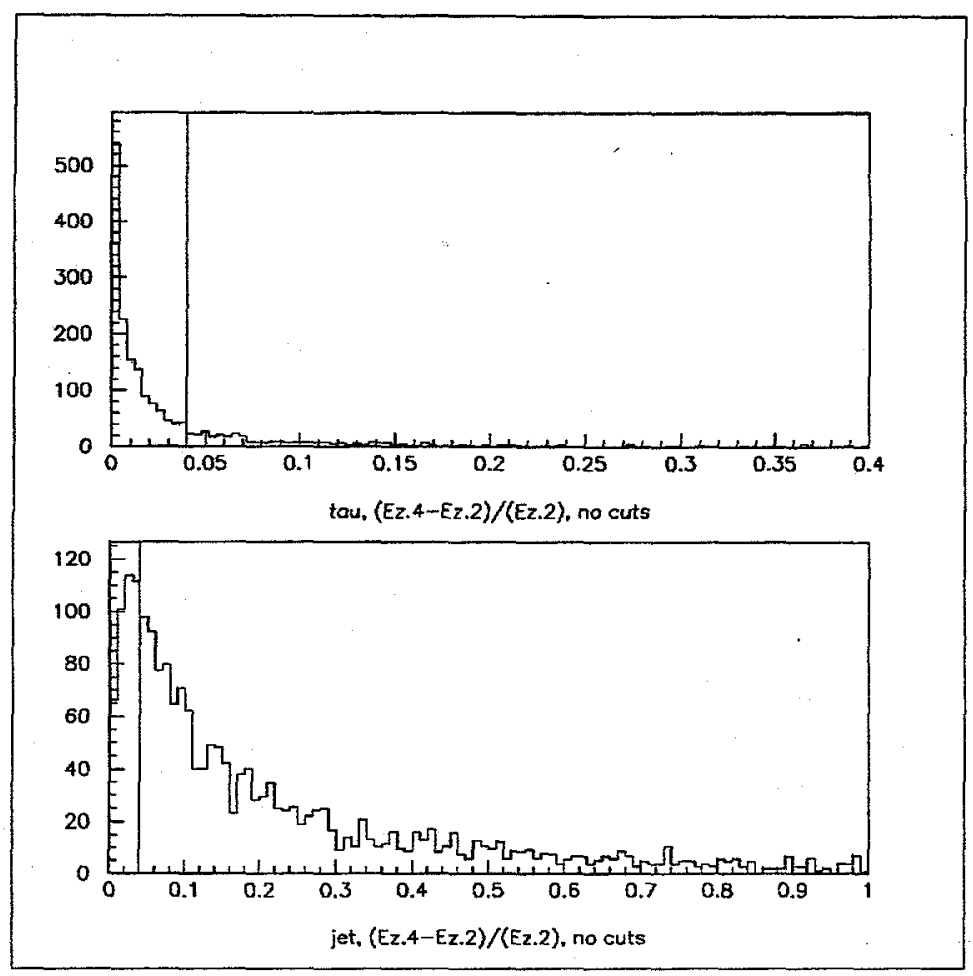

Figure 1: The quantity $I$ as defined in the text for hadronic tau decays (top) and jets (bottom). Clearly, the taus are wider than the jets. The line indicates the cut at .04, clearly this cut eliminates most of the jets without eliminating many taus. The samples of events with $M_{Z}=91 \mathrm{GeV}$ are used.

obtained. The efficiency for accepting hadronic tau decays and the corresponding rejection factor against jets is shown in Table 1 . An additional rejection can be obtained by studying the mass of the jet, calculated by assuming that the energy in each calorimeter cell is due to a single massless particle at the center of the cell, as a function of the transverse energy of the jet. Figure 2 shows a scatterplot of these variables. It can be seen that the QCD jets have larger mass than the jets from tau decay. This to be expected because, up to the effects of the detector and the presence of particles inside the jet cone not from tau itself, the mass of the tau decay product is limited by the tau mass while the mass of QCD jets should increase with transverse energy. After applying the cut at $I_{0}=0.04$ many QCD jets, particularly those with larger masses are rejected. If an additional cut rejecting events with $\frac{m}{p_{t}}>0.065$ or $m>3.6 \mathrm{GeV}$ is applied, the rejection against QCD jets can be improved as is illustrated by the lowest pair of figures in Figure 2.

Combining the cut at $I_{0}=0.04$ with the calorimetric mass cut gives a rejection against jets of 15 and an efficiency for hadronic tau decays of $65 \%$. This rejection factor is sufficient for the supersymmetry cases of interest ${ }^{\ddagger}$ This is because cuts on missing transverse energy

\footnotetext{
${ }^{\ddagger}$ A larger rejection can be obtained by using the total measured calorimeter energy. the same cuts give
} 


\begin{tabular}{|c|c|c|}
\hline$I_{0}$ & efficiency \% & rejection \\
\hline \hline .01 & 9.84 & 34.58 \\
.02 & 59.91 & 13.68 \\
.03 & 67.88 & 8.15 \\
.04 & 73.98 & 5.83 \\
.05 & 77.27 & 4.66 \\
.06 & 79.98 & 3.93 \\
.07 & 82.54 & 3.46 \\
.08 & 84.06 & 3.09 \\
.09 & 85.39 & 2.84 \\
.10 & 86.23 & 2.61 \\
\hline
\end{tabular}

Table 1: Efficiencies in percent for acceptance of taus and rejection factors for jets as a function of $I_{0}$ for the $M_{Z}=91 \mathrm{GeV}$ sample.

and the presence of energetic jets [3] is sufficient to reject standard model background and to obtain an event sample dominated by supersymmetry events. In this case the rejection that is achieved by our tau cuts must be sufficient to discriminate against additional QCD jets in the supersymmetry events. Since the multiplicity of these jets is small, the rejection that we obtained is adequate.

\section{Further selection of tau events and the tau-tau mass distribution}

In order to make a detailed study of the taus we need to exploit the ATLAS tracking system to measure the momentum of charged tracks and the finely segmented electromagnetic calorimeter to measure the energy and hence the momentum of the photons that arise from $\pi^{0}$ decay. The events are first passed through the calorimeter cuts described in the previous section. Insufficient QCD jets pass those cuts so that further detailed study of their properties is not possible and the remainder of this section is therefore dedicated to the hadronic tau candidates only. In this section a default cone size of $\Delta R=0.2$ is used.

For jets that are in the fiducial tracking region $-2.5<\eta<2.5$, the Xkalman routine is used to reconstruct tracks in a road defined either by the direction of the tau (as determined from the event generator) or of the jet resulting from the tau decay products reconstructed in the calorimeter. The results from the two choices are equivalent. These reconstructed tracks are then extrapolated to the electromagnetic calorimeter, assuming first a constant magnetic field ending abruptly at a radius of $1.2 \mathrm{~m}^{\S}$, and then a straight line extrapolation up to the EM shower maximum radius (assumed independent of $p_{\perp}$ ). Tracks are included if they hit the calorimeter inside the cone of the same radius as that used to find the jets

a rejection factor of 82 and for $32 \%$ efficiency, when $I$ is computed from the full calorimeter.

${ }^{\S}$ This corresponds to the model used in DICE 


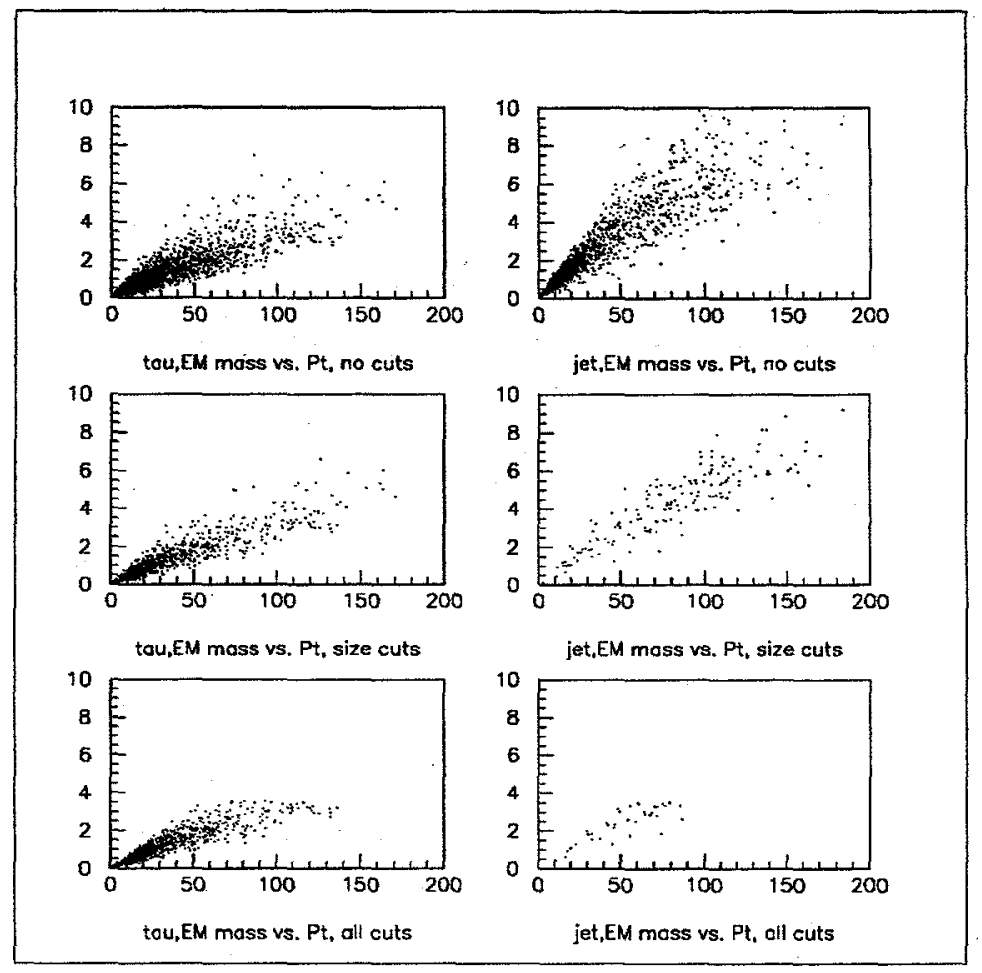

Figure 2: The transverse momentum of a jet as a function of its mass, where both quantities are determined from the electromagnetic calorimeter alone. The plots on the left correspond to hadronic tau decays while the plots on the right correspond to jets. The first row shows the plots for no cuts applied, the second with only the $I_{0}=0.04$ cuts applied and the third with all the cuts. The samples of events with $M_{Z}=91 \mathrm{GeV}$ are used.

and centered on the core of the jet. A $\chi^{2}$ variable using the reconstructed track parameters and the track parameters of the particles from the tau decay (taken from the generator) is exploited to determine whether a reconstructed track comes from a $\tau$. Such tracks are referred to as matched tracks. All other tracks (so called unmatched tracks) are real extra particles. 'All tracks' refers to both matched and unmatched tracks. There are two source of unmatched tracks; conversions of photons in the tracker and minimum bias particles, mainly pions, that happen to be close to the tau direction.

The identity of the tracks with transverse momentum greater than $500 \mathrm{MeV}(1 \mathrm{GeV})$ is shown in Figure $3(4)$. Using these figures to compare the "all tracks" and "matched tracks" shows two differences. There are very few electrons in the "matched track" sample".

\footnotetext{
ITracks reconstructed by XKalman are identified by pointing to tracks in the KINE bank of DICE. Low $p_{t}$ electrons that result from conversions are not tagged in the KINE bank. In this case the reconstructed tracks are associated with the parent photon. This accounts for the "photons" shown in Figures 3 and 4. These are actually electron tracks. We are grateful to D. Barberis and D. Rousseau for information on this point.
} 
These can be traced to rare tau decays. The extra electrons and positrons in the "all tracks" sample are due to conversions. The additional number of pions is due to the underlying event particles. The fact that the latter are non-negligible implies that the methodology suggested in this paper cannot be extended trivially to the case of high luminosity. The presence of these additional tracks means that the mass of the "tau" that we will determine next can exceed the true tau mass even in the case of perfect detector resolution.

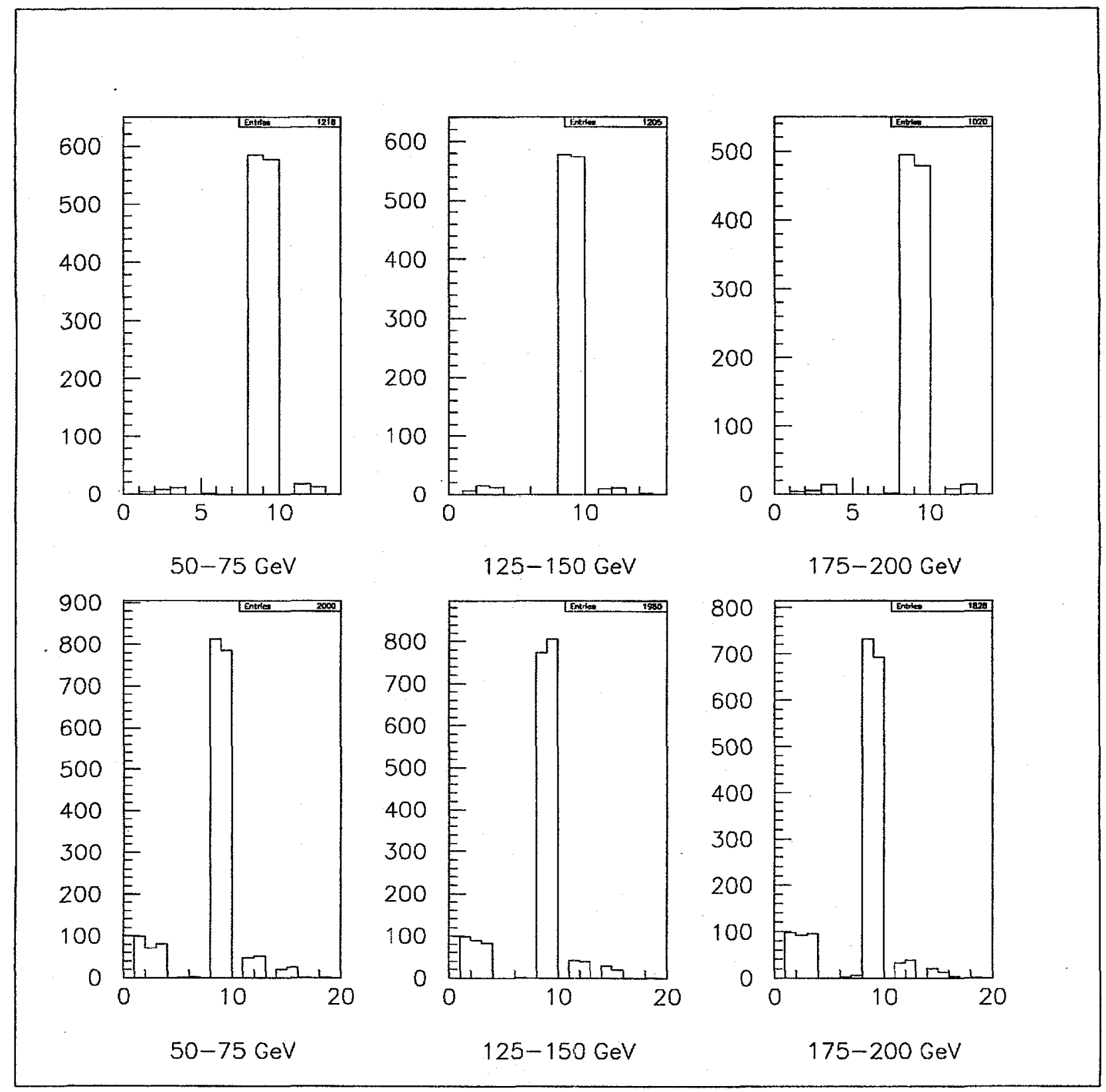

Figure 3: Particle GEANT identification number $\left(2: e^{+}, 3: e^{-}, 8: \pi^{+}, 9: \pi^{-}\right)$. Matched tracks on the top row, all tracks on the bottom row.

The presence of these additional tracks implies that the multiplicity of charged tracks observed will differ from that in tau decays. The $\tau$ decay channels are almost exclusively 

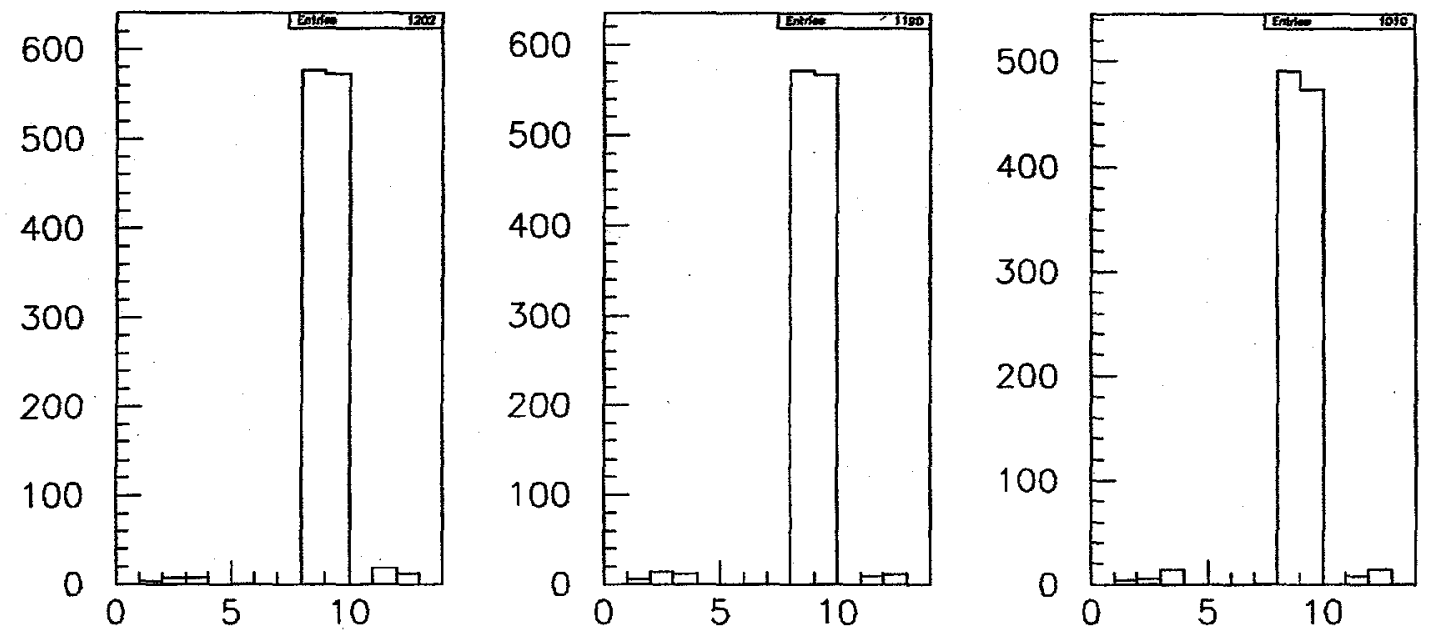

$50-75 \mathrm{GeV}$
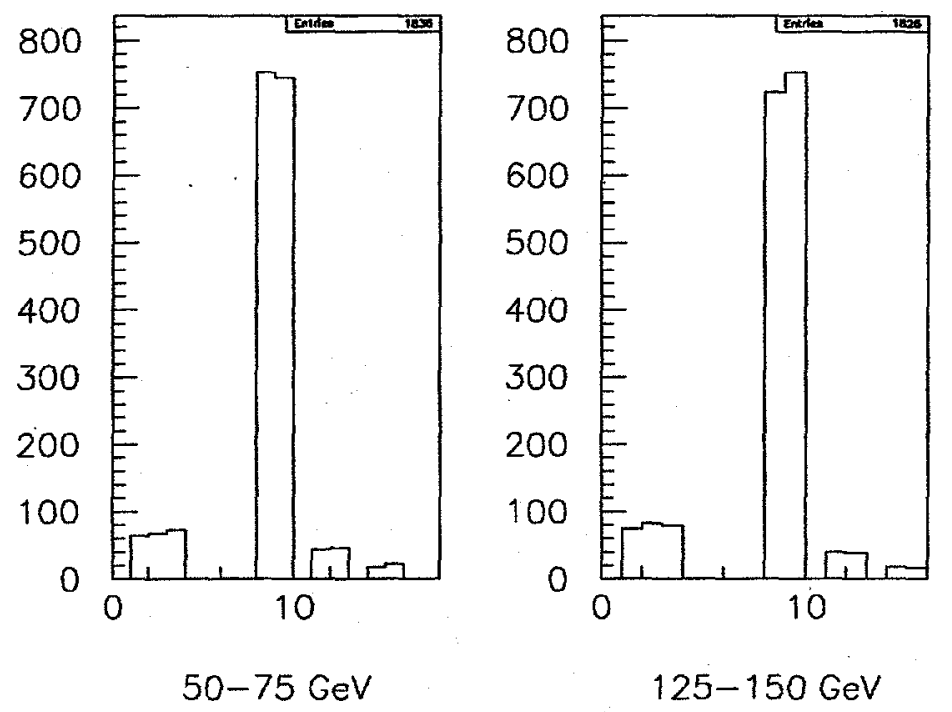

$175-200 \mathrm{GeV}$

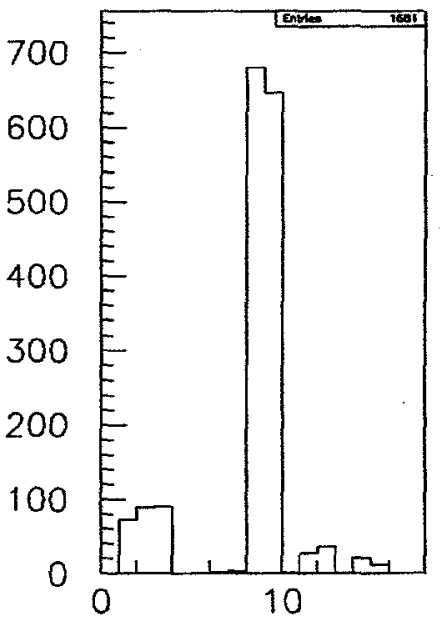

$175-200 \mathrm{GeV}$

Figure 4: Particle GEANT identification number $\left(2: e^{+}, 3: e^{-}, 8: \pi^{+}, 9: \pi^{-}\right)$. Matched tracks on the top row, all tracks on the bottom row, $1 \mathrm{GeV}$ cut.

1-prong and 3-prong. The number of tracks should therefore be one or three. As shown on Figures $5 \& 6$ this is not exactly the case: around $6.8 \%$ (resp. $7.4 \%$ for the $1 \mathrm{GeV}$ case) of the jets contain two matched tracks. In addition to the effect of additional tracks, low $p_{t}$ tracks can be swept outside of the cone and poorly reconstructed tracks lost. The presence of reconstructed taus with an even number of tracks complicates the ability to determine the charge of the tau. This will be discussed further below.

We can now form the mass of a single tau jet as follows. All electromagnetic cells with $E_{t}>1 \mathrm{GeV}$ (i.e. the same cut as on the charged tracks) were assumed to have a massless 


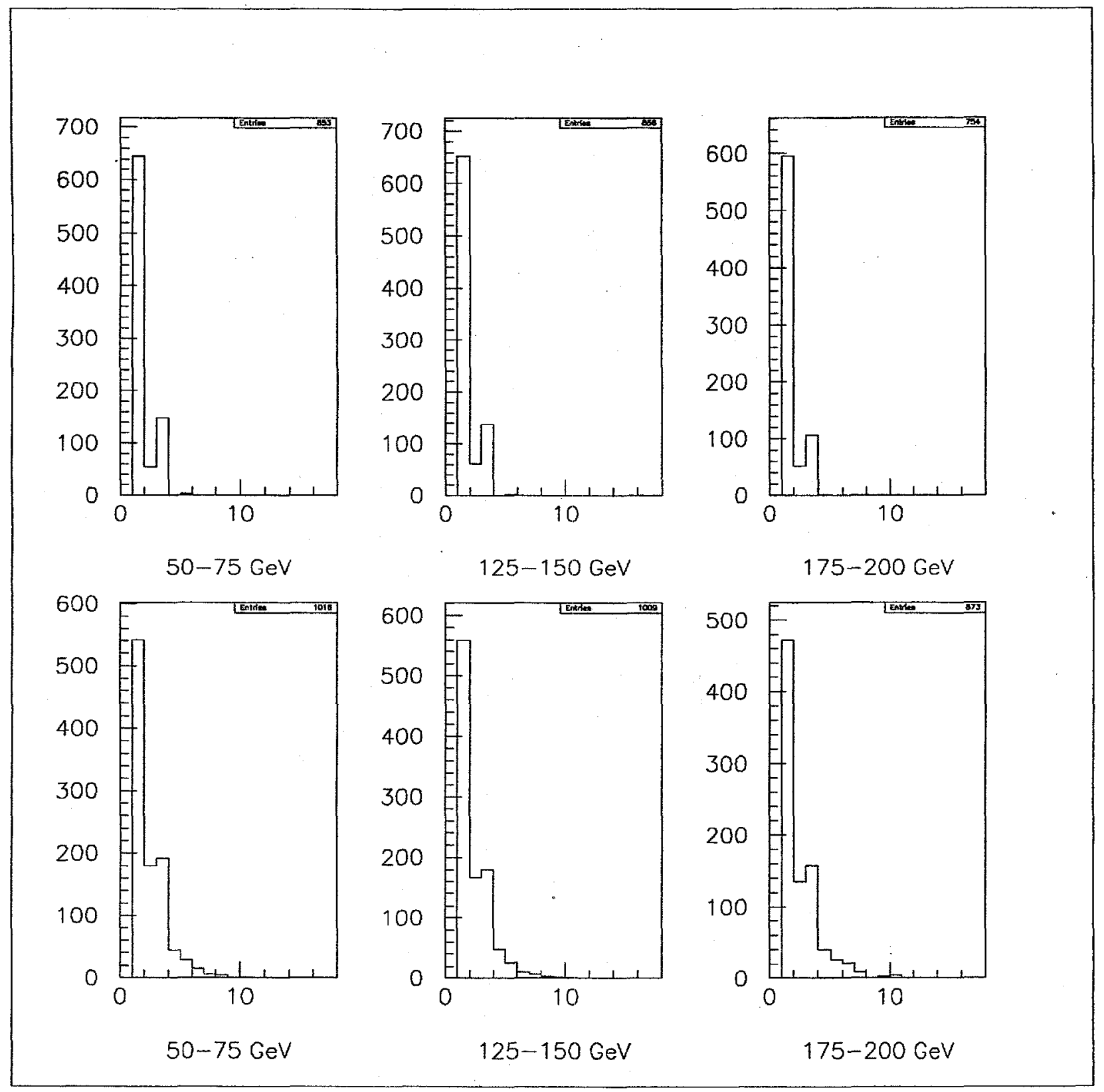

Figure 5: Number of reconstructed tracks per jet. Matched tracks on the top row, all tracks on the bottom row. 


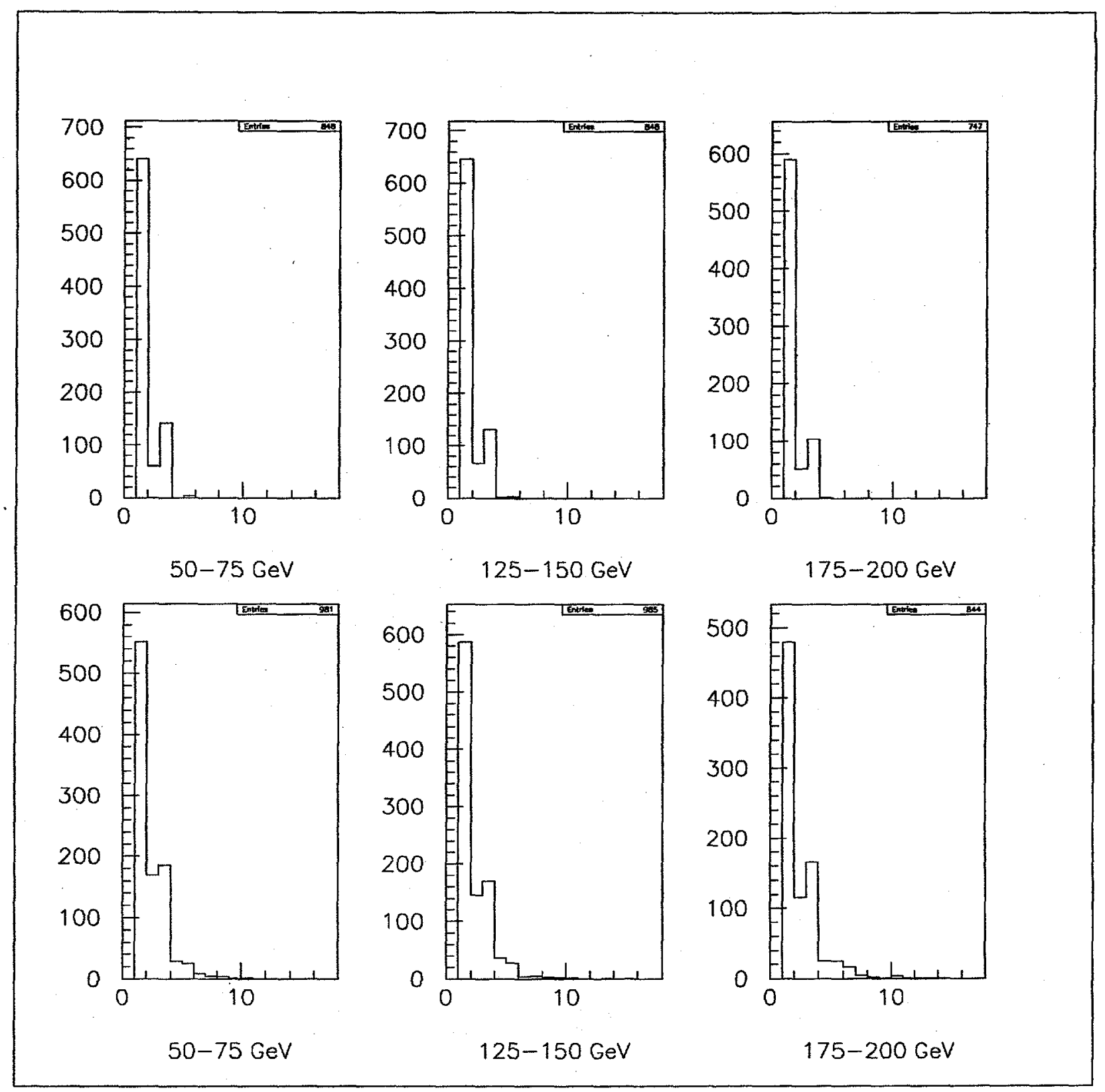

Figure 6: Number of reconstructed tracks per jet. Matched tracks on the top row, all tracks on the bottom row, $1 \mathrm{GeV}$ cut. 
particle at their center with an energy corresponding to the measured energy in that cell. These "particles" can then be combined with the reconstructed charged tracks, which are all assumed to be pions, to determine the jet mass. The hadron calorimeter is not used. If a charged track hits a calorimeter cell and deposits energy, we must be careful to avoid over counting. If the track is not an electron, the calorimeter energy is presumed to be due to an early shower and is not included when computing the mass. If the track is an electron, then we keep the electromagnetic energy and do not include the track. We are assuming that it will prove possible to identify these conversion electrons. The shower spread from an early showering charged pion is such that it can deposit energy in cells adjacent to the one that is hit. However, we cannot reject neighboring cells without loosing significant tau energy as the jets from tau decay are very narrow. A two dimensional event display is shown in Figure 7. This event has two photons and one charged track $(\tau \rightarrow \rho \nu)$; the tracks has no electromagnetic calorimeter energy in the cell that it passes through and the photons hit neighboring cells with 8 and $14 \mathrm{GeV}$ deposited.

The reconstructed mass distribution is shown in Figure 8 . This can be compared with the mass spectrum shown from the generated particles (not including neutrinos) shown in Figure 9. The basic structure is still present after detector effects are included. The most significant detector effect is the spreading of events beyond the tau mass. This has two causes; resolution effects and extra particles caused by conversions and underlying event particles. The resolution effect gets worse as the transverse momentum of the produced $Z$, and hence the tau momentum, increases and the track measurements become more imprecise. This effect can be seen clearly from the "matched track" plots in Figure 8. The second effect can be seen by comparing the distributions of "all tracks" and "matched tracks" in the same plot. The additional high mass tail in the latter case is due to contributions to the tau invariant mass from invariant mass of a tau decay product and a underlying event particle. This effect also increases with the transverse momentum of the tau. If the cut on the transverse momentum of tracks is increased to $1 \mathrm{GeV}$ the resulting tau mass is shown in figure 10

For events where there are two jets each having reconstructed tracks from a tau, we can compute the observed mass of the jet-jet system. This is shown in Figure 11. A broad distribution is obtained. If a cut is applied requiring that each tau have a reconstructed mass greater than $0.8 \mathrm{GeV}$, the tau-tau mass distribution has a sharper peak and larger mean as can be see in Figure 12. The effect is simple to understand, as the cut is increased the neutrino carries less energy and therefore more of the tau energy is observed Table 2 shows this effect in detail by showing the variation of the width and mean of the tau-tau mass distribution as the cut on the reconstructed tau mass is increased. The cut at $M_{\tau}=0.8$ retains approximately $40 \%$ of the tau pair events. The reconstructed tau-tau mass peaks are slightly narrower if the the individual tracks and calorimeter cells are required to have $P_{t}>1 \mathrm{GeV}$ as can be seen by comparing Figures 12 and 13 . For this reason adopt this stiffer cut as the default.

Before we can parametrise the results of our study, we must investigate the dependence on the generated tau-tau mass (i.e. the $Z$ mass) and the transverse momentum. From Table 3 it can be seen that the dependence on the generated mass of the ratio of the peak position and width to the generated mass is small. The ratio of peak position to generated mass is slightly higher at the lowest value of $M_{Z}$. The relative importance of the underlying events increases as $M_{Z}$ is reduced and could account for some of this increase. The statistical significance 


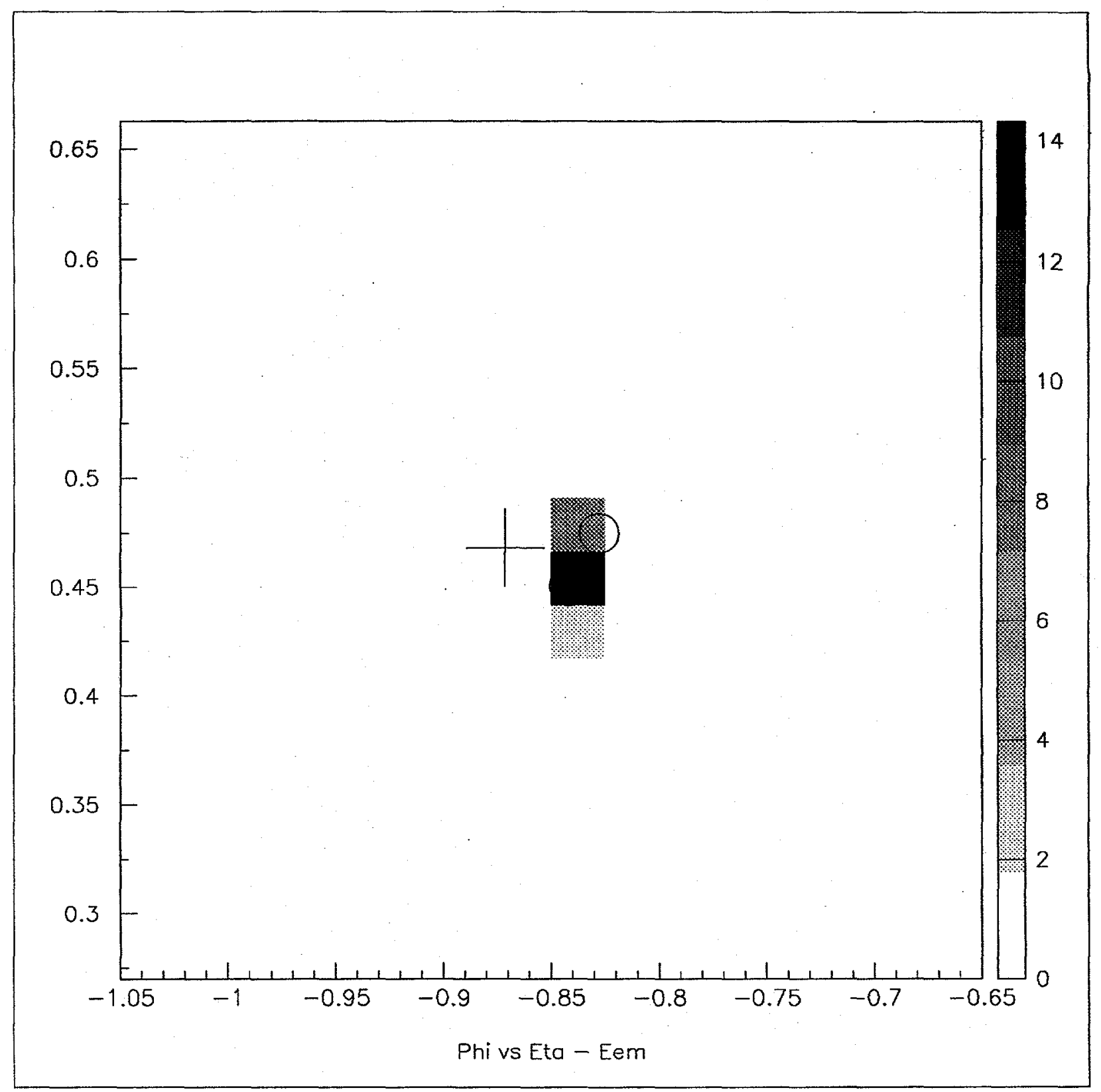

Figure 7: The energy deposits in the electromagnetic calorimeter in $\mathrm{GeV}$ (colored/intensity scale) on the $\phi$ (ordinate) $\eta$ (abscissa) plane from a tau decay. The extrapolated position of the tau decay products is shown for charged particles (crosses) and photons (open circles). One of the two photons is in the center of the highest energy cell. 


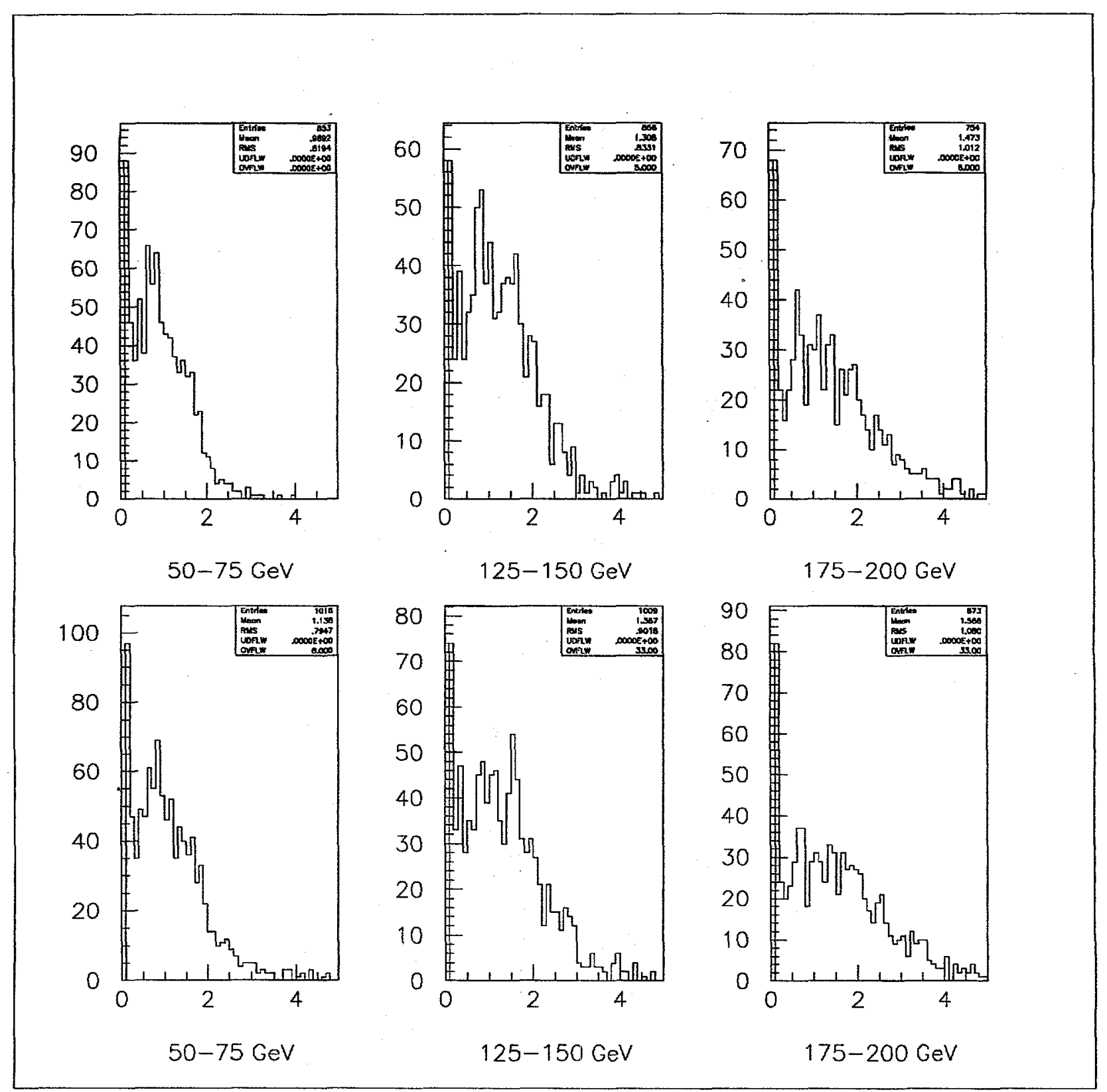

Figure 8: Reconstructed $\tau$ invariant mass. Matched tracks on the top row, all tracks on the bottom row. 


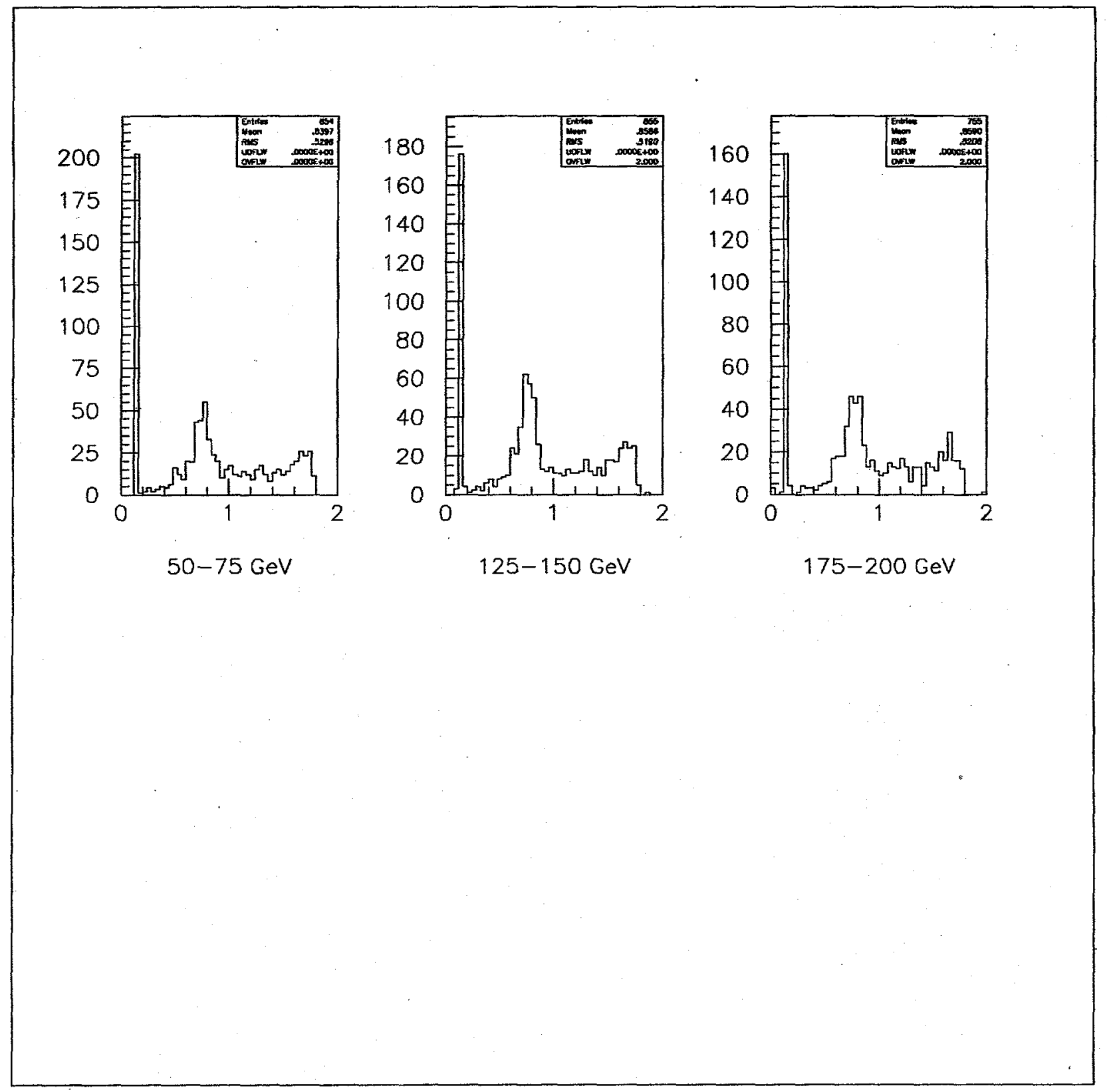

Figure 9: $\tau$ invariant mass from the generated tracks. 

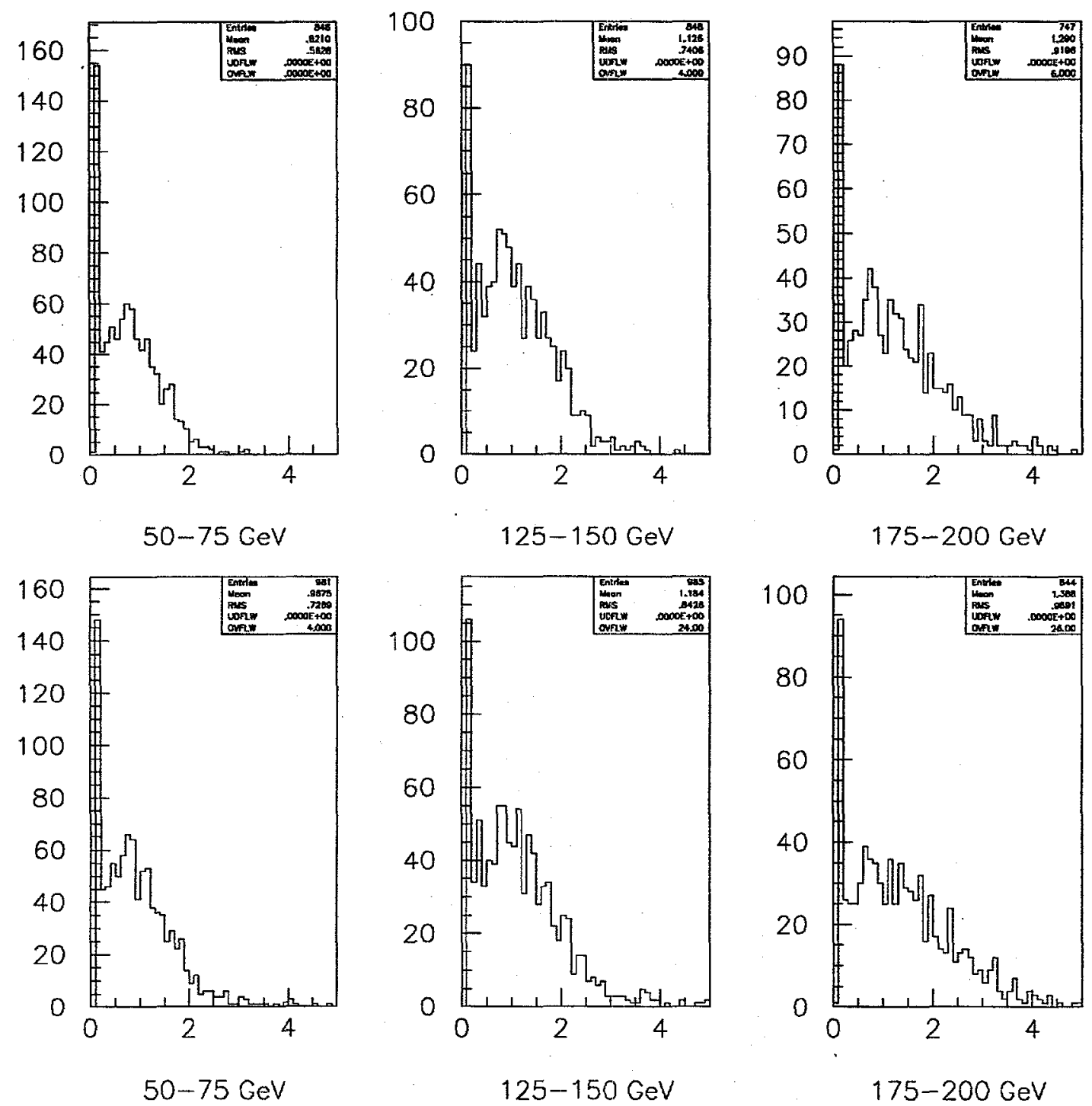

Figure 10: $\tau$ invariant mass. Matched tracks on the top row, all tracks on the bottom row, $1 \mathrm{GeV}$ cut. 
of these differences is not great; our data samples are rather small and have large statistical uncertainties. The reconstruction efficiency is not strongly dependent up the generated mass as the table also shows. Examination of Figure 11 shows that the dependence on the transverse momentum of the $Z$ is also small.
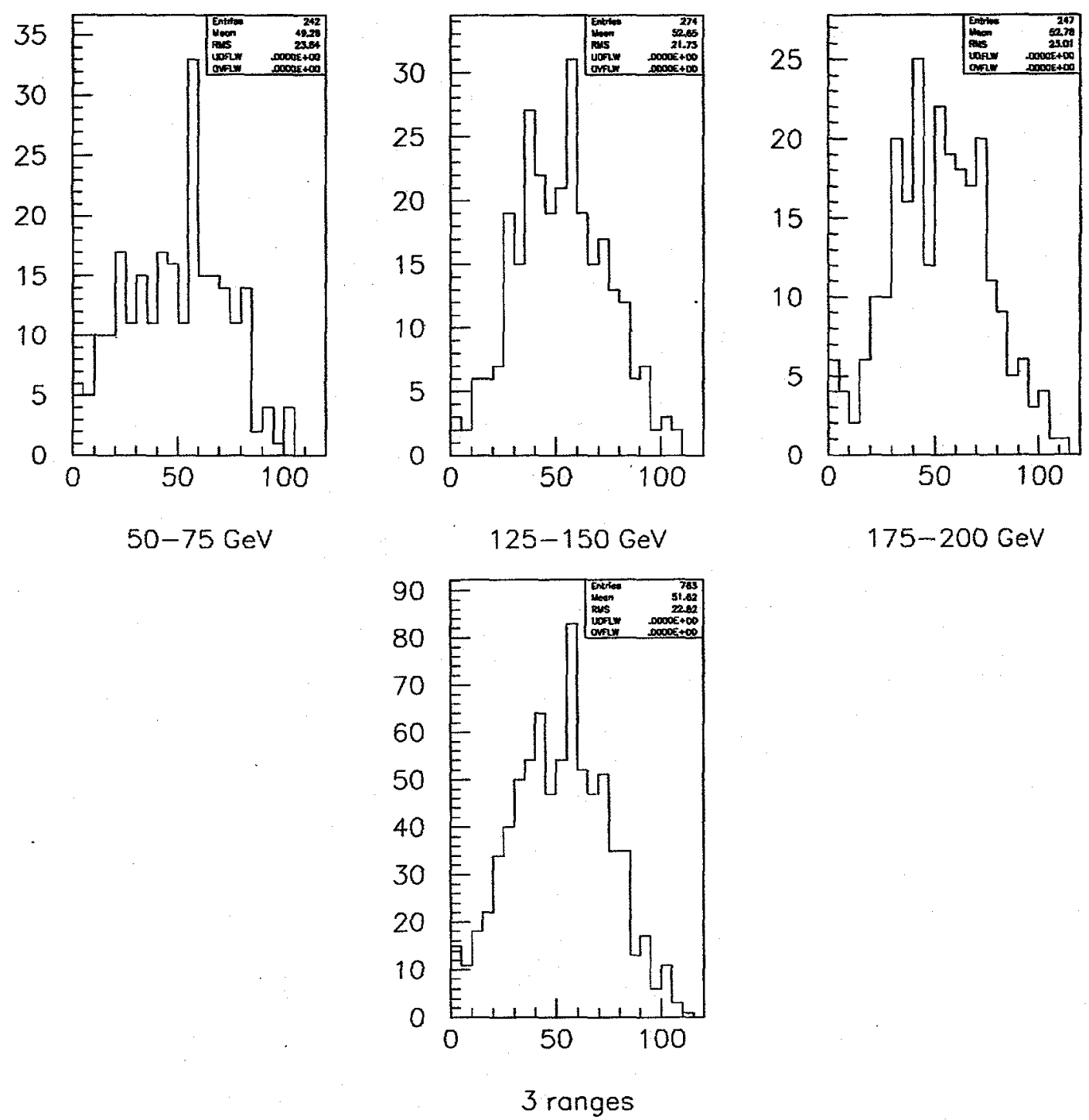

Figure 11: Invariant mass of the tau-tau system, all tracks. The last plot is the sum of the three others.

Finally we consider the measurement of the charge of the hadronically decaying tau. The supersymmetry events can have several taus in them; we are looking for structure in the $\tau^{+} \tau^{-}$invariant mass distributions. If we are not able to identify charges, the distribution will be compromised by the featureless $t a u^{+} \tau^{+}$distribution. Since there are extra tracks within 

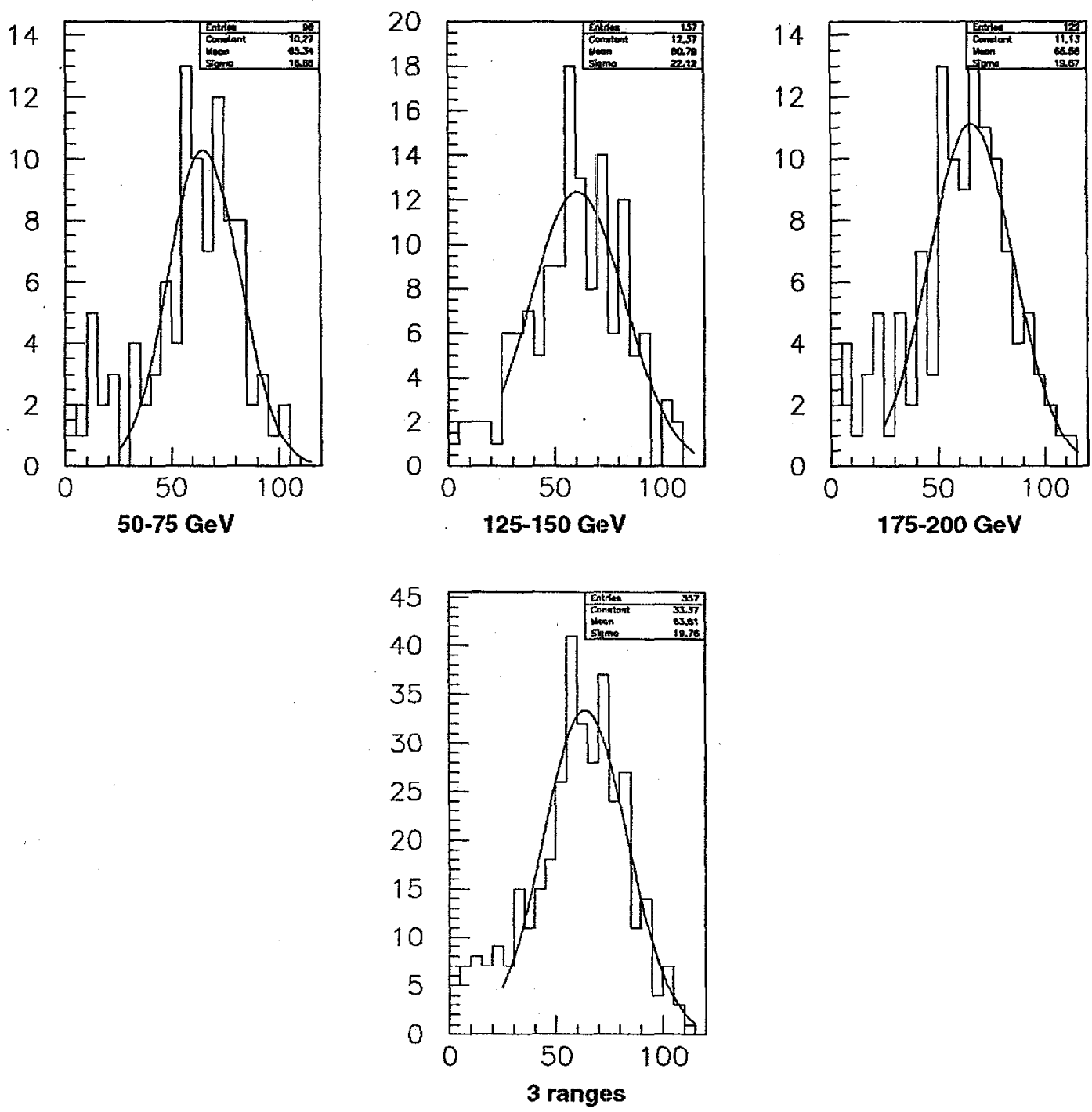

Figure 12: tau-tau invariant mass, all tracks, $m_{\tau}>0.8 \mathrm{GeV}$. The last plot is the sum of the three others. 


\begin{tabular}{|l|l|l|}
\hline$M_{\tau}>$ & Mean & Sigma \\
\hline \hline nocut & $45.42 \pm 2.36$ & $26.18 \pm 1.98$ \\
0.8 & $65.54 \pm 2.11$ & $16.29 \pm 2.23$ \\
0.85 & $66.80 \pm 1.92$ & $15.04 \pm 1.78$ \\
0.9 & $68.47 \pm 2.11$ & $14.45 \pm 2.20$ \\
0.95 & $71.21 \pm 1.93$ & $12.50 \pm 2.49$ \\
1.0 & $71.44 \pm 1.94$ & $12.34 \pm 2.26$ \\
1.05 & $72.15 \pm 2.09$ & $12.62 \pm 2.39$ \\
1.1 & $72.27 \pm 2.37$ & $13.55 \pm 2.70$ \\
1.15 & $71.10 \pm 2.46$ & $12.98 \pm 3.40$ \\
1.2 & $74.45 \pm 3.19$ & $14.81 \pm 4.14$ \\
\hline
\end{tabular}

Table 2: tau-tau invariant mass: Mean and sigma of the distribution for different cuts on $\mathrm{m}_{\tau}$, with $50<p_{\perp}<75 \mathrm{GeV}$.

\begin{tabular}{|l|l|l|l|l|l|}
\hline$M_{Z}$ & Mean & Sigma & Mean $/ M_{Z}$ & Sigma $/ M_{Z}$ & Efficiency \\
\hline \hline 25. & 18.6 & 4.9 & 0.75 & 0.2 & 0.42 \\
50. & 36.3 & 8.9 & 0.72 & 0.18 & 0.47 \\
75. & 50.0 & 12.4 & 0.66 & 0.165 & 0.42 \\
91. & 63 & 17.5 & 0.67 & 0.192 & 0.38 \\
200. & 131 & 38 & 0.65 & 0.19 & 0.54 \\
\hline
\end{tabular}

Table 3: Mean and sigma of the reconstructed tau-tau mass distribution for different values of $M_{Z}$. The event samples have $125 \mathrm{Gev} c<P_{T}(Z)<150 \mathrm{GeV}$. The ratios shown in the last three columns are independent of $M_{Z}$ within the errors in the fit. 


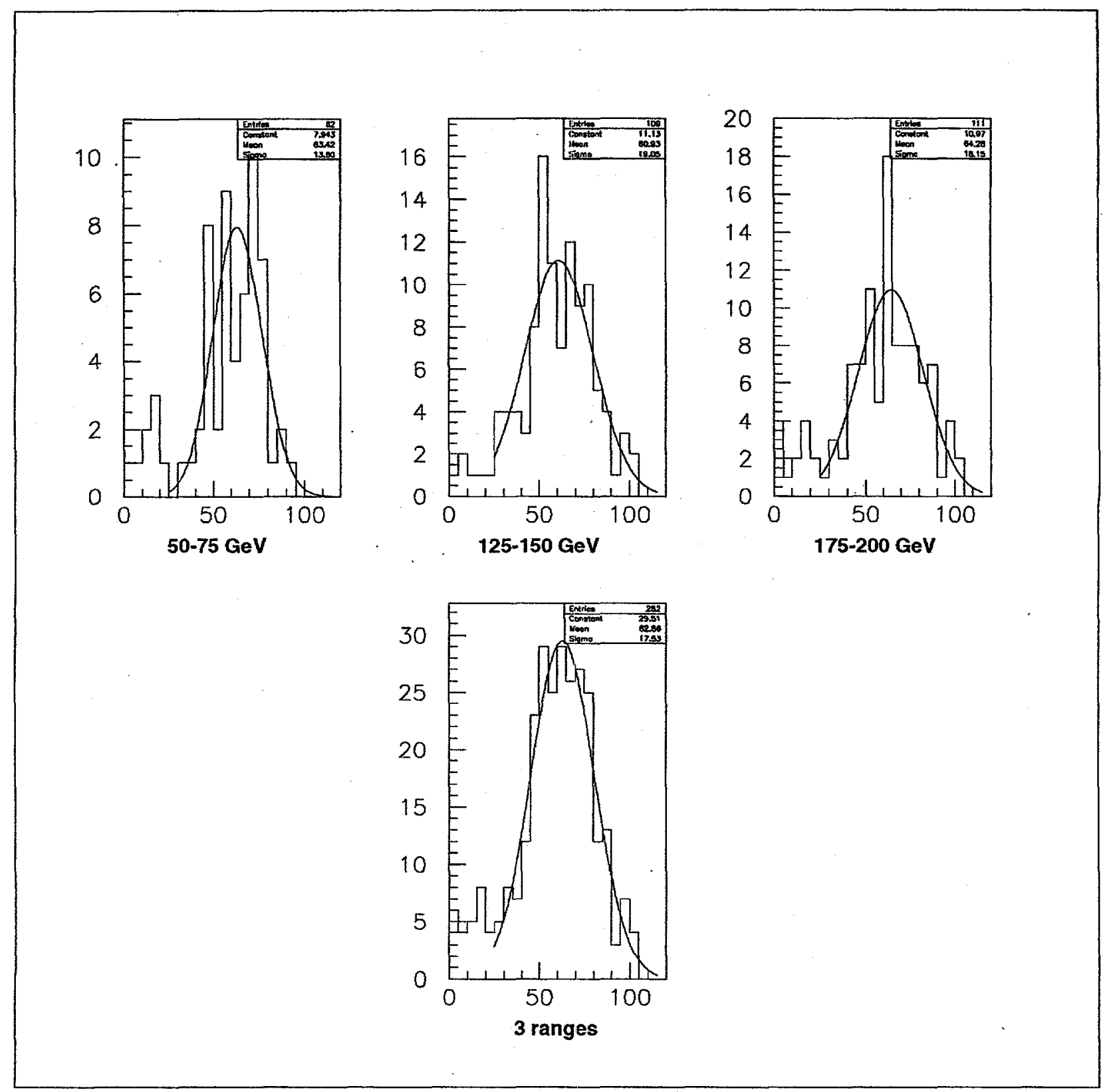

Figure 13: tau-tau invariant mass, all tracks, $m_{\tau}>0.8 \mathrm{GeV}$. The last plot is the sum of the three others. Individual tracks and calorimeter cells are now required to have $P_{t}>1 \mathrm{GeV}$. 
the reconstruction cone, from underlying event and conversions, and some slow tracks from the tau can be lost, this measurement is not fully efficient. If the sign total charge of the tracks within the cone is used, $75 \%$ of the taus are signed correctly. Of the remainder $21 \%$ have no net charge and the rest are identified incorrectly. A better result can be obtained by increasing the cone size to $\Delta R=0.4$ and using the sign of the weighted jet charge defined by

$$
\sum_{i}\left|p_{i}\right| q_{i}
$$

where $q_{i}$ is the charge of a particle of momentum $p_{i}$. This enables more of the tau decay products to the included and reduces the impact of the (slower) underlying event particles. Using this criterion $92 \%$ of the taus are correctly assigned.

\section{Parameterization of Results}

The cuts described above correspond to an efficiency for each hadronically decaying $\tau$ of $65 \%$ for the isolation cuts and $63 \%$ for the reconstructed tau mass cut, giving a combined efficiency of $41 \%$. The charge of the tau is correctly determined in $92 \%$ of the cases. The rejection factor for jets is 15 from the isolation cuts. We have insufficient statistics to determine the additional rejection that would arise from the reconstructed tau mass cut, so, conservatively we assume that all of the jets pass this. The charge is not determined for these fake taus. The reconstructed tau-tau mass distribution can be reproduced, within our errors, by a central value of $M_{\tau \tau}^{\text {expt }}=0.66 M_{\tau \tau}^{\text {generated }}$ and a resolution of $\sigma=0.18 M_{\tau}^{\text {generated }}$ The effect of this resolution on the tau tau mass distribution expected in supersymmetry events will now be shown.

Events were generated using the SUGRA [7] model as implemented in ISAJET-7.37 [8] with $m_{0}=m_{1 / 2}=200 \mathrm{GeV}, A_{0}=0, \tan \beta=45$ and $\mu<0$. These events are passed through the simulation described in [3]. Events are then selected by requiring at least 4 hadronic jets (some of which could be from the hadronic decay of a tau). All four of these jets must have $p_{T}>50 \mathrm{GeV}$ and at least one of them must have $p_{t}>100 \mathrm{GeV}$. The scalar sum of the transverse energies of the four largest $p_{t}$ jets and the missing transverse momentum is formed $\left(E_{s}\right)$. The missing transverse momentum must be greater than the larger of $100 \mathrm{GeV}$ and $0.2 E_{S}$. These stringent cuts are sufficient to reduce the standard model background to a negligible level. The hadronic taus are now selected as follows. Jets are searched for with $p_{t}>20 \mathrm{GeV}$ and $|\eta|<2.5$. If there is a generated tau within $\Delta R=0.4$ of this jet, the jet is associated with the tau. The generated tau momenta are then used with the above parameterization to simulate the $\tau-\tau$ mass distribution. Jets that are not associated with taus are candidate for "fake taus". The rejection factor above is applied and the resulting jet marked. The background from events where both taus are fake is negligible. The invariant mass distribution of tau-"fake-tau" is made by using the measured energy of the two jets in question, together with the tau efficiency factors. Figure 14 shows the reconstructed tau-tau mass distribution for tau pairs that have opposite charge. This plot shows a peak in the distribution which is a clear signal of the sharp edge in the generated tau-tau mass distribution that occurs at $59.6 \mathrm{GeV}$ : The shape and position of this peak can be used to determine the location of the edge. The background from fake taus is shown separately on 
the figure and is small. More details of this analysis will be presented elsewhere [9].

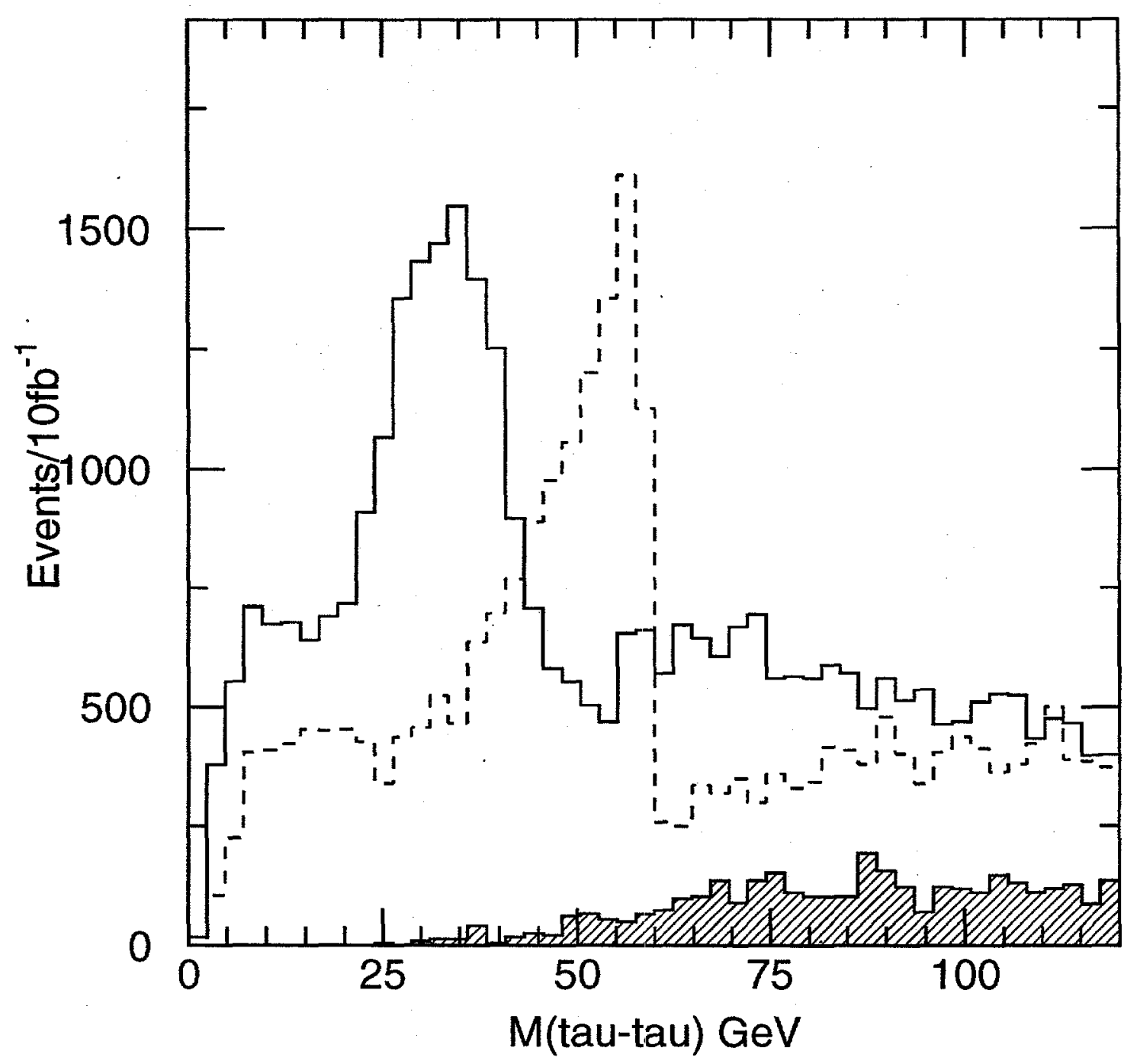

Figure 14: The distribution of tau-tau invariant mass in supersymmetry events resulting from the parameterization developed in this note. The taus are required to have opposite charges. The solid histogram shows the reconstructed tau-tau invariant mass using the selection criteria and parameterization given in the text. The dashed line shows the shape of the generated tau-tau mass distribution with an edge at $59.6 \mathrm{GeV}$. This plot has been scaled by the reconstruction efficiency to facilitate comparison. The filled histogram shows the contribution from combinations formed by a real tau and a jet that fakes a tau. 


\section{Conclusion}

In this note, we have demonstrated how hadronic tau decays can be reconstructed in the ATLAS detector. At the cost of lowering the rejection factor against light quark jets, we are able to reconstruct hadronic tau decays quite efficiently. We measure the mass of the hadronic jet by combining the momenta of charged tracks with the energy measured in the electromagnetic calorimeter. By cutting on this quantity, we can select tau decays where the observed decay products carry a large fraction of the tau's energy. We can then the reconstruct the tau-tau invariant mass and obtain better resolution than would be the case if all hadronic tau's were selected. In particular the resolution is much better than one would obtain if only the $\tau \rightarrow \pi \nu$ mode were used.

This study has application in the study of supersymmetry at the LHC, where, for certain choices of parameters, the decays of supersymmetric particles lead to final states with pairs of taus, and the ability to measure the tau-tau invariant mass leads to a precise measurement of a mass difference between supersymmetric particles. The results from our full simulation study are parameterized for use in fast simulation.

Acknowlegments The computing necessary for this work was carried out on the PDSF facility operated by NERSC. We are grateful for their support. The work was supported in part by the Director, Office of Energy Research, Office of High Energy Physics, Division of High Energy Physics of the U.S. Department of Energy under Contract DE-AC0376SF00098. Accordingly, the U.S. Government retains a nonexclusive, royalty-free license to publish or reproduce the published form of this contribution, or allow others to do so, for U.S. Government purposes. 


\section{References}

[1] D. Cavalli et.al. Atlas Internal Note PHYS-No-051.

[2] D. Cavalli and S. Resconi Atlas Internal Note PHYS-No-118.

[3] I. Hinchliffe, et.al. Phys. Rev. D55, 3520 (1997).

[4]

http://atlasinfo.cern.ch/Atlas/GROUPS/SOFTWARE/DOCUMENTS/DICE_310/dice310.html

[5] G. Polesello, et.al. Atlas Internal Note PHYS-No-111.

[6] T. Sjostrand, Comput.Phys.Commun.82:74-90,1994

[7] L. Alvarez-Gaume, J. Polchinski and M.B. Wise, Nucl. Phys. B221, 495 (1983);

L. Ibañez, Phys. Lett. 118B, 73 (1982);

J.Ellis, D.V. Nanopolous and K. Tamvakis, Phys. Lett. 121B, 123 (1983);

K. Inoue et al. Prog. Theor. Phys. 68, 927 (1982);

A.H. Chamseddine, R. Arnowitt, and P. Nath, Phys. Rev. Lett., 49, 970 (1982). For reviews see, H.P. Nilles, Phys. Rep. 111, 1 (1984);

H.E. Haber and G.L. Kane, Phys. Rep. 117, 75 (1985).

[8] F. Paige and S. Protopopescu, in Supercollider Physics, p. 41, ed. D. Soper (World Scientific, 1986);

H. Baer, F. Paige, S. Protopopescu and X. Tata, in Proceedings of the Workshop on Physics at Current Accelerators and Supercolliders, ed. J. Hewett, A. White and D. Zeppenfeld, (Argonne National Laboratory, 1993).

[9] In preparation. 